\title{
Hausdorff measure of noncompactness of matrix operators on some sequence spaces of a double sequential band matrix
}

\author{
Elahe Abyar and Mohammad Bagher Ghaemi*
}

\section{"Correspondence:}

mghaemi@iust.ac.ir

Islamic Azad University, Karaj

Branch, Alborz, Iran

\begin{abstract}
The sequence spaces $I_{\infty}(\tilde{B}, p), c(\tilde{B}, p)$, and $c_{0}(\tilde{B}, p)$ of non-absolute type derived by the double sequential band matrix $B(\tilde{r}, \tilde{s})$ have recently been defined. In this work, we establish identities or estimates for the operator norms and the Hausdorff measure of noncompactness of certain matrix operators on these spaces that are paranormed spaces. Further, we find the necessary and sufficient condition for compactness of $L_{A}$ in the class $\left(X, I_{\infty}(q)\right)$ (where $X$ is any of the spaces $I_{\infty}(\tilde{B}, p), c(\tilde{B}, p)$ or $\left.c_{0}(\tilde{B}, p)\right)$ and characterize some classes of compact operators on these spaces by using the Hausdorff measure of the noncompactness technique.
\end{abstract}

Keywords: Hausdorff measure of noncompactness; double sequential matrix; sequence space; paranormed space

\section{Preliminaries and background}

The first measure of noncompactness, the function $\alpha$, was defined and studied by Kuratowsky [1] in 1930. Darbo [2], using this measure, generalized both the classical Schauder fixed point principle and (a special variant of) Banach's contraction mapping principle for so called condensing operators. The Hausdorff measure of noncompactness $\chi$ was introduced by Goldenstein et al. [3] in 1957.

Recently, the Hausdorff measure of noncompactness turned out to be very useful in the classification of compact operators between Banach spaces. Many authors characterized the classes of compact operators given by infinite matrices on some sequence spaces by using the Hausdorff measure of noncompactness. For example, in [4, 5] Malkowsky and Djolovic, in [6, 7] Malkowsky and Rakocevic, in [8] Alotaibi et al., in [9] Basar and Malkowsky, and in $[10,11]$ Mursaleen and Noman have applied the Hausdorff measure of noncompactness to characterize some classes of compact operators given by matrices on the spaces in the literature.

Further, as we know paranormed spaces are another version of the linear metric space but have more general properties than normed spaces [12]. Hence, some authors have applied these spaces in their research. For example, in [13] Basarir and Kara have characterized some classes of compact operators given by matrices on a normed sequence space, which is a special case of the paranormed Riesz $B^{m}$-difference sequence space $r^{q}\left(p, B^{m}\right)$

(c) 2015 Abyar and Ghaemi. This article is distributed under the terms of the Creative Commons Attribution 4.0 International License (http://creativecommons.org/licenses/by/4.0/), which permits unrestricted use, distribution, and reproduction in any medium, provided you give appropriate credit to the original author(s) and the source, provide a link to the Creative Commons license, and indicate if changes were made. 
and for this purpose they have applied the Hausdorff measure of noncompactness. In $[14,15]$ Basarir and Kara and in [16] Ozger and Basar have studied these spaces.

In [17] Kirisci and Basar have studied the domain of generalized difference matrix $B(r, s)$ in the classical spaces $l_{\infty}, c$, and $c_{0}$.

Afterward, Ozger and Basar in [16] introduced the paranormed sequence spaces $l_{\infty}(\tilde{B}, p), c(\tilde{B}, p)$, and $c_{0}(\tilde{B}, p)$ that are more general and comprehensive than the corresponding consequences of the matrix domain of $B(r, s)$.

In this work, we establish identities or estimates for the operator norms and the Hausdorff measure of noncompactness of certain matrix operators on these spaces that were defined by Ozger and Basar in [16].

Let $w$ be the space of all real or complex valued sequences. Any vector subspace of $w$ is called a sequence space. We write $l_{\infty}, c$ and $c_{0}$ for the spaces of all bounded, convergent, and null sequences, respectively. Also, by $b s, c s, l_{1}$, and $l_{p}(1<p<\infty)$, we denote the spaces of all bounded, convergent, absolutely, and $p$-absolutely convergent series, respectively. A sequence space $\lambda$ with a linear topology is called a $K$-space if each of the maps $P_{i}$ : $\lambda \rightarrow \mathbb{C}$ defined by $P_{i}(x)=x_{i}$ is continuous for all $i \in \mathbb{N}$, where $\mathbb{C}$ is the set of all complex numbers. A $K$-space $\lambda$ is called an $F K$-space provided $\lambda$ is a complete linear metric space and a $B K$-space if it is a normed $F K$-space. Let $\phi$ be the set of all sequences which have finite number of non-zero terms. An $F K$-space $\lambda$ which contains $\phi$ is said to have the $A K$ property if every sequence $x=\left(x_{k}\right)_{k=0}^{\infty} \in \lambda$ has a unique representation $x=\sum_{k=0}^{\infty} x_{k} e^{(k)}[18]$.

A linear topological space $X$ over the real field $\mathbb{R}$ is said to be a paranormed space if there is a subadditive function $h: X \rightarrow \mathbb{R}$ such that $h(\theta)=0, h(-x)=h(x)$, and scalar multiplication is continuous, that is, $\left|\alpha_{n}-\alpha\right| \rightarrow 0$ and $h\left(x_{n}-x\right) \rightarrow 0$ imply $h\left(\alpha_{n} x_{n}-\alpha x\right) \rightarrow 0$ for every $\alpha \in \mathbb{R}$ and $x \in X$, where $\theta$ is the zero vector in the linear space $X$ [19].

Throughout this paper, we assume $\left(p_{k}\right)$ is a bounded sequence of strictly positive real numbers with $\sup p_{k}=H$ and $M=\max \{1, H\}$. So, the sequence spaces $\left.l_{\infty}(p), c_{(} p\right), c_{0}(p)$, and $l(p)$ (which generalize the classical spaces $l_{\infty}, c, c_{0}$, and $l_{1}$, respectively) are defined as follows:

$$
\begin{aligned}
& l_{\infty}(p)=\left\{x=\left(x_{k}\right) \in w: \sup _{k \in \mathbb{N}}\left|x_{k}\right|^{p_{k}}<\infty\right\}, \\
& c(p)=\left\{x=\left(x_{k}\right) \in w: \exists l \in \mathbb{C} \ni \lim _{k \rightarrow \infty}\left|x_{k}-l\right|^{p_{k}}=0\right\}, \\
& c_{0}(p)=\left\{x=\left(x_{k}\right) \in w: \lim _{k \rightarrow \infty}\left|x_{k}\right|^{p_{k}}=0\right\}, \\
& l(p)=\left\{x=\left(x_{k}\right) \in w: \sum_{k}\left|x_{k}\right|^{p_{k}}<\infty\right\} \quad\left(0<p_{k}<\infty\right) .
\end{aligned}
$$

Let the functions $h_{1}$ and $h_{2}$ be defined on the spaces $l_{\infty}(p), c(p)$ or $c_{0}(p)$, and $l(p)$ by

$$
h_{1}(x)=\sup _{k \in \mathbb{N}}\left|x_{k}\right|^{\frac{p_{k}}{M}} \quad \text { and } \quad h_{2}(x)=\left(\sum_{k}\left|x_{k}\right|^{p_{k}}\right)^{\frac{1}{M}} .
$$

The sequence spaces $c_{0}(p)$ and $c(p)$ are complete paranormed spaces paranormed by $h_{1}$ if $p \in l_{\infty}$ ([20], Theorem 6). $l_{\infty}(p)$ is also complete paranormed space by $h_{1}$ if and only if $p_{k}>0$, and also $l(p)$ is complete paranormed space paranormed by $h_{2}$ and $\left\{e^{(k)}\right\}_{k \in \mathbb{N}}$ is a basis in the space $l(p)$. 
The domain of an infinite matrix $A$ in a sequence space $\lambda$ is denoted by $\lambda_{A}$ where

$$
\lambda_{A}=\left\{x=\left(x_{k}\right) \in w: A x \in \lambda\right\} .
$$

It is obvious that $\lambda_{A}$ is a sequence space.

Let $(X,\|\cdot\|)$ be a normed space and $X \supset \phi$ be a $B K$-space and $a=\left(a_{k}\right) \in w$, then the $\alpha$-, $\beta$-, and $\gamma$-dual of a subset $X$ of $w$ are, respectively, defined by

$$
\begin{aligned}
& X^{\alpha}=\left\{a=\left(a_{k}\right) \in w: a x=\left(a_{k} x_{k}\right) \in l_{1} \text { for all } x=\left(x_{k}\right) \in X\right\}, \\
& X^{\beta}=\left\{a=\left(a_{k}\right) \in w: a x=\left(a_{k} x_{k}\right) \in c s \text { for all } x=\left(x_{k}\right) \in X\right\}, \\
& X^{\gamma}=\left\{a=\left(a_{k}\right) \in w: a x=\left(a_{k} x_{k}\right) \in b s \text { for all } x=\left(x_{k}\right) \in X\right\} .
\end{aligned}
$$

If $A$ is an infinite matrix with complex entries $a_{n k}(n, k \in \mathbb{N})$, then we write $A=\left(a_{n k}\right)$. We define the $A$-transform of $x$ as the sequence $A x=\left(A_{n}(x)\right)_{n=0}^{\infty}$, where

$$
A_{n}(x)=\sum_{k=0}^{\infty} a_{n k} x_{k} \quad(n \in \mathbb{N}),
$$

if $x=\left(x_{k}\right) \in w$ and provided the series on the right-hand side converges for each $n \in \mathbb{N}$.

If $X$ and $Y$ are subsets of $w$ and $A=\left(a_{n k}\right)$ is an infinite matrix, then $A$ defines a matrix mapping from $X$ into $Y$, and we denote it by $A: X \rightarrow Y$, if $A x$ exists and is in $Y$ for all $x \in X$. We denote the class of all infinite matrices that map $X$ into $Y$ by $(X, Y)$. So, $A \in(X, Y)$ if and only if $A_{n} \in X^{\beta}$ for all $x \in X$ (we write $A_{n}$ for the sequence in the $n$th row of $A$, i.e., $A_{n}=\left(a_{n k}\right)_{k=0}^{\infty}$ for every $n \in \mathbb{N}[21]$.

The following results are fundamental for our work.

\section{Remark 1.1}

(a) ([22]) Let $\dagger$ denote any of the symbols $\alpha, \beta$ or $\gamma$. Then we have $c_{0}^{\dagger}=c^{\dagger}=l_{\infty}^{\dagger}=l_{1}$, $l_{1}^{\dagger}=l_{\infty}$, and $l_{p}^{\dagger}=l_{q}$ where $1<p<\infty$ and $q=p /(p-1)$.

(b) ([21]) Let $X$ be any of the spaces $c_{0}, c, l_{\infty}$ or $l_{p}(1 \leq p<\infty)$. Then $\|\cdot\|_{X^{\beta}}$ denotes the natural norm on the dual space $X^{\beta}$.

(c) ([23]) Let $X \supset \phi$ and $Y$ be a $B K$-space. Then we have the following:

(c') $(X, Y) \subset B(X, Y)$, that is, every matrix $A \in(X, Y)$ defines an operator $L_{A} \in B(X$, $Y$ ) by $L_{A}(x)=A x$ for all $x \in X$.

(c') If $X$ has $A K$, then $B(X, Y) \subset(X, Y)$, that is, for every operator $L_{A} \in B(X, Y)$ there exists a matrix $A \in(X, Y)$ such that $L_{A}(x)=A x$ for all $x \in X$.

Remark 1.2 ([10]) Let $X \supset \phi$ be a $B K$-space. Then:

(a) If $Y$ is any of the spaces $c_{0}, c$ or $l_{\infty}$, and $A \in(X, Y)$, then

$$
\left\|L_{A}\right\|=\|A\|_{\left(X, l_{\infty}\right)}=\sup _{n}\left\|A_{n}\right\|_{X}^{*}<\infty .
$$

(b) If $A \in\left(X, l_{1}\right)$, then

$$
\|A\|_{\left(X, l_{1}\right)} \leq\left\|L_{A}\right\| \leq 4 \cdot\|A\|_{\left(X, l_{1}\right)},
$$


where

$$
\|A\|_{\left(X, l_{1}\right)}=\sup _{N \in F}\left\|\sum_{n \in \mathbb{N}} A_{n}\right\|_{X}^{*}<\infty
$$

where $F$ denotes the collection of all finite subsets of $\mathbb{N}$, and $F_{r}(r \in \mathbb{N})$ is the subcollection of $F$ consisting of all nonempty subsets of $\mathbb{N}$ with elements grater than $r$, that is,

$$
F_{r}=\{N \in F: n>r \text { for all } n \in \mathbb{N}\} \quad(r \in \mathbb{N}) .
$$

\section{The sequence spaces $I_{\infty}(\tilde{B}, p), c(\tilde{B}, p)$, and $c_{0}(\tilde{B}, p)$}

In this section we define the sequence spaces of non-absolute type that derived by the double sequential band matrix $B(\tilde{r}, \tilde{s})$. These sequence spaces are the complete paranormed linear spaces.

Let $k, n \in \mathbb{N}$ and $\tilde{r}=\left(r_{k}\right)$, and $\tilde{s}=\left(s_{k}\right)$ be the convergent sequences whose entries are either constant or distinct non-zero numbers. Then we define the double sequential matrix $B(\tilde{r}, \tilde{s})=\left\{b_{n k}\left(r_{k}, s_{k}\right)\right\}_{n, k=0}^{\infty}$ by

$$
b_{n k}= \begin{cases}r_{k}, & k=n, \\ s_{k}, & k=n-1, \\ 0, & \text { otherwise }\end{cases}
$$

Recently, Ozger and Basar [16] introduced the sequence spaces $l_{\infty}(\tilde{B}, p), c(\tilde{B}, p)$, and $c_{0}(\tilde{B}, p)$ as follows:

$$
\begin{aligned}
& l_{\infty}(\tilde{B}, p)=\left\{x=\left(x_{k}\right) \in w: \sup _{k \in \mathbb{N}}\left|r_{k} x_{k}+s_{k-1} x_{k-1}\right|^{p_{k}}<\infty\right\}, \\
& c(\tilde{B}, p)=\left\{x=\left(x_{k}\right) \in w: \exists l \in \mathbb{C} \ni \lim _{k \rightarrow \infty}\left|r_{k} x_{k}+s_{k-1} x_{k-1}-l\right|^{p_{k}}=0\right\}, \\
& c_{0}(\tilde{B}, p)=\left\{x=\left(x_{k}\right) \in w: \lim _{k \rightarrow \infty}\left|r_{k} x_{k}+s_{k-1} x_{k-1}\right|^{p_{k}}=0\right\},
\end{aligned}
$$

and the $B(\tilde{r}, \tilde{s})$-transforms of these spaces are in the spaces $l_{\infty}(p), c(p)$ and $c_{0}(p)$, respectively.

Because of the notation (1) we have

$$
l_{\infty}(\tilde{B}, p)=\left[l_{\infty}(p)\right]_{\tilde{B}}, \quad c(\tilde{B}, p)=[c(p)]_{\tilde{B}} \quad \text { and } \quad c_{0}(\tilde{B}, p)=\left[c_{0}(p)\right]_{\tilde{B}} .
$$

Throughout, we define the sequence $y=\left(y_{k}\right)$ by the $B(\tilde{r}, \tilde{s})$-transform of a sequence $x=\left(x_{k}\right)$, that is,

$$
y_{k}=\{B(\tilde{r}, \tilde{s}) x\}_{k}=r_{k} x_{k}+s_{k-1} x_{k-1}, \quad \forall k \in \mathbb{N}
$$

Since the spaces $\lambda_{(\tilde{B}, p)}$ and $\lambda$ are norm isomorphic for any sequence space $\lambda$, we observe that $x=\left(x_{k}\right) \in \lambda_{(\tilde{B}, p)}$ if and only if $y=\left(y_{k}\right) \in \lambda$, where the sequences $x=\left(x_{k}\right)$ and $y=\left(y_{k}\right)$ are 
connected by (2). Let $(X,\|\cdot\|)$ be a normed space. If $X \supset \phi$ is a $B K$-space and $a=\left(a_{k}\right) \in w$ then we write

$$
\|a\|_{X}^{*}=\sup _{x \in S_{X}}\left|\sum_{k=0}^{\infty} a_{k} x_{k}\right|,
$$

provided the expression on the right-hand side exists and is finite.

Now, we have the following result.

Lemma 2.1 The sequence spaces $l_{\infty}(\tilde{B}, p), c(\tilde{B}, p)$, and $c_{0}(\tilde{B}, p)$ are $B K$-spaces with the same paranorm given by

$$
\|x\|_{l_{\infty}(\tilde{B}, p)}=\|B(\tilde{r}, \tilde{s})(x)\|_{l_{\infty}(p)}=\sup _{k \in \mathbb{N}}\left|B_{k}(\tilde{r}, \tilde{s})(x)\right|^{\frac{p_{k}}{M}}
$$

and they are linearly isomorphic to the spaces $l_{\infty}(p), c(p)$, and $c_{0}(p)$.

Proof See Theorem 3.1 in [24].

Now, we have the following lemma by the previous result.

Lemma 2.2 Let $X$ denote any of the spaces $l_{\infty}(\tilde{B}, p), c(\tilde{B}, p)$ or $c_{0}(\tilde{B}, p)$. If $a=\left(a_{k}\right) \in X^{\beta}$, then $\tilde{a}=\left(\tilde{a}_{k}\right) \in l(p)$ and the equality

$$
\sum_{k=0}^{\infty} a_{k} x_{k}=\sum_{k=0}^{\infty} \tilde{a}_{k} y_{k}
$$

holds for every $x=\left(x_{k}\right) \in X$, where $y=B(\tilde{r}, \tilde{s})(x)$ is the associated sequence defined by (2) and

$$
\tilde{a}_{k}=\sum_{m=0}^{k} \frac{(-1)^{k-m}}{r_{k}} \prod_{j=m}^{k-1} \frac{s_{j}}{r_{j}} a_{n} .
$$

Proof The equality (5) derived by using equation (2) from the $m$ th partial sum of the series $\sum_{k} a_{n k} x_{k}$, so

$$
\sum_{k=0}^{m} a_{n k} x_{k}=\sum_{k=0}^{m-1} \tilde{a}_{n k} y_{k}+\left(\sum_{k=m}^{\infty} \frac{(-1)^{m-k}}{r_{m}} \prod_{j=k}^{m-1} \frac{s_{j}}{r_{j}} a_{n j}\right) y_{m},
$$

that the summation running from 0 to $m-1$ is equal to zero when $m=0$. Now, when $m \rightarrow \infty$ we obtain

$$
\sum_{k} a_{k} x_{k}=\sum_{k} \tilde{a}_{k} y_{k}, \quad \forall n \in \mathbb{N} .
$$

Lemma 2.3 If $X$ denotes any of the spaces $l_{\infty}(\tilde{B}, p), c(\tilde{B}, p)$ or $c_{0}(\tilde{B}, p)$, then

$$
\|a\|_{X}^{*}=\|\tilde{a}\|_{X^{\beta}}=\|\tilde{a}\|_{l(p)}=\left(\sum_{k=0}^{\infty}\left|\tilde{a}_{k}\right|^{p_{k}}\right)^{\frac{1}{M}}<\infty,
$$

where $a=\left(a_{k}\right) \in X^{\beta}$ and $\tilde{a}=\left(\tilde{a}_{k}\right)$ is a sequence defined by (6). 
Proof Let $a=\left(a_{k}\right) \in X^{\beta}$. Then, by using Lemma 2.2 we have $\tilde{a}=\left(\tilde{a}_{k}\right) \in l(p)$ and $y=y_{k} \in Y$ (we assume that $Y$ are, respectively, the spaces $l_{\infty}(p), c(p)$, and $c_{0}(p)$ ), which are connected by equation (2). Further, it follows by (4) that $x \in S_{X}$ if and only if $y \in S_{Y}$. Therefore, we derive from (3) and (5) that

$$
\|a\|_{X}^{*}=\sup _{x \in S_{X}}\left|\sum_{k=0}^{\infty} a_{k} x_{k}\right|=\sup _{y \in S_{Y}}\left|\sum_{k=0}^{\infty} \tilde{a}_{k} y_{k}\right|=\|\tilde{a}\|_{Y}^{*},
$$

and since $\tilde{a} \in l(p)$, we obtain from Remark 1.1 (parts (a) and (b))

$$
\|a\|_{X}^{*}=\|\tilde{a}\|_{Y}^{*}=\|\tilde{a}\|_{l(p)}<\infty
$$

This completes the proof.

Lemma 2.4 Let $X$ be any of the spaces $l_{\infty}(\tilde{B}, p), c(\tilde{B}, p)$ or $c_{0}(\tilde{B}, p)$, and $Y$ be, respectively, the spaces $l_{\infty}(p), c(p)$ or $c_{0}(p)$ and $V$ be a sequence space and $A=\left(a_{n k}\right)$ be an infinite matrix. If $A \in(X, V)$, then $\tilde{A} \in(Y, V)$ such that $A x=\tilde{A} y$ for all sequences $x \in X$ and $y \in Y$ which are connected by equation (2), where $\tilde{A}=\left(\tilde{a}_{n k}\right)$ is the associated matrix defined by

$$
\tilde{a}_{n k}=\sum_{i=k}^{\infty} \frac{(-1)^{i}}{r_{i}} \prod_{j=k}^{i-1} \frac{s_{j}}{r_{j}} a_{n i} \quad(\forall n \in \mathbb{N}) .
$$

Proof Let $x \in X$ and $y \in Y$ be connected by equation (2) and suppose that $A \in(X, V)$. Then, by using Lemma 2.3, we obtain $\tilde{A}_{n} \in l(p)=X^{\beta}$ for all $n \in N$ and the equality $A x=\tilde{A} y$ holds, hence $\tilde{A} y \in V$. Since every $y \in Y$ is the associated sequence of some $x \in X$, we deduce that $\tilde{A} \in(Y, V)$. This completes the proof.

Theorem 2.1 Let $A=\left(a_{n k}\right)$ be an infinite matrix and $\tilde{A}=\left(\tilde{a}_{n k}\right)$ be the associated matrix defined by (11) and let $X$ be any of the spaces $l_{\infty}(\tilde{B}, p), c(\tilde{B}, p)$ or $c_{0}(\tilde{B}, p)$. If $A$ is in any of the classes $\left(X, c_{0}(p)\right),(X, c(p))$ or $\left(X, l_{\infty}(p)\right)$, then

$$
\left\|L_{A}\right\|=\|A\|_{\left(X, l_{\infty}(p)\right)}=\sup _{n \in \mathbb{N}}\left(\sum_{n=0}^{\infty}\left|\tilde{a}_{n k}\right|^{p_{k}}\right)^{\frac{1}{M}}<\infty .
$$

Proof By combining Remark 1.2(a) and Lemma 2.3, we obtain the equality in (12).

\section{The Hausdorff measure of noncompactness}

Recently, many authors characterized the classes of compact operators given by infinite matrices on some sequence spaces by using the Hausdorff measure of noncompactness. For example, Mursaleen and Noman in [18] introduced the notion of generalized means and studied some topological properties of the spaces of generalized means. In [21] they have characterized some matrix operators on these spaces by applying the Hausdorff measure of noncompactness. Further, Mursaleen and Noman in [10, 25, 26], Basarir and Kara in [13, 27], Mursaleen et al. in [28], Kara and Basarir in [29], Maji and Srivastava in [30], Kara et al. in [31] and Alotaibi et al. in [23] characterized some classes of compact operators on the spaces in the literature by using the Hausdorff measure of the noncompactness method. 
Let $(X,\|\cdot\|)$ be a normed space. Then the unit sphere and the closed unit ball in $X$ are denoted by $S_{X}=\{x \in X:\|x\|=1\}, \bar{B}_{X}=\{x \in X:\|x\| \leq 1\}$. For the Banach spaces $X$ and $Y$ we denote the set of all bounded linear operators $L: X \rightarrow Y$ by $B(X, Y)$ with the operator norm given by $\|L\|=\sup _{x \in S_{X}}\|L(x)\|$. A linear operator $L: X \rightarrow Y$ is said to be compact if the domain of $L$ is all of $X$ and, for every bounded sequence $\left(x_{n}\right)$ in $X$, the sequence $L\left(x_{n}\right)$ has a subsequence which converges in $Y$. We denote the class of all compact operators in $B(X, Y)$ by $C(X, Y)$. An operator $L \in B(X, Y)$ is said to be of finite rank if $\operatorname{dim} R(L)<\infty$, where $R(L)$ denotes the range of $L$. An operator of finite rank is clearly compact [32].

Let $S$ and $M$ be subsets of a metric space $(X, d)$ and $\epsilon>0$. Then $S$ is called an $\epsilon$-net of $M$ in $X$ if for every $x \in M$ there exists $s \in S$ such that $d(x, s)<\epsilon$. If the set $S$ is finite, then the $\epsilon$-net $S$ of $M$ is called a finite $\epsilon$-net of $M$, and we say that $M$ has a finite $\epsilon$-net in $X$. A subset of a metric space is said to be totally bounded if it has a finite $\epsilon$-net for every $\epsilon>0$ [10].

We denote the collection of all bounded subsets of a metric space $(X, d)$ by $M_{X}$. If $Q \in$ $M_{X}$, then the Hausdorff measure of noncompactness of the set $Q$, denoted by $\chi(Q)$, is defined by

$$
\chi(Q)=\inf \{\epsilon>0: Q \text { has a finite } \epsilon \text {-net in } X\} .
$$

The function $\chi: M_{X} \rightarrow[0, \infty)$ is called the Hausdorff measure of noncompactness.

The basic properties of the Hausdorff measure of noncompactness can be found in [22, $33,34]$. For example, if $Q, Q_{1}$, and $Q_{2}$ are bounded subsets of a metric space $(X, d)$, then:

(1) $\chi(Q)=0$ if and only if $Q$ is totally bounded,

(2) $Q_{1} \subset Q_{2}$ implies $\chi\left(Q_{1}\right) \leq \chi\left(Q_{2}\right)$.

Further, if $X$ is a normed space, then the function $\chi$ has some additional properties connected with the linear structure, that is,

(1) $\chi\left(Q_{1}+Q_{2}\right) \leq \chi\left(Q_{1}\right)+\chi\left(Q_{2}\right)$

(2) $\chi(\alpha Q)=|\alpha| \chi(Q), \forall \alpha \in \mathbb{C}$.

Let $X$ and $Y$ be Banach spaces and $L \in B(X, Y)$. Then the Hausdorff measure of noncompactness of $L$, denoted by $\|L\|_{\chi}$, is defined by

$$
\|L\|_{\chi}=\chi\left(L\left(S_{X}\right)\right)
$$

and

$$
\|L\|_{\chi}=0 \quad \text { if and only if } L \in C(X, Y) \quad \text { [33]. }
$$

The following theorem gives an estimate for the Hausdorff measure of noncompactness in Banach spaces with Schauder bases and in this theorem $I$ denotes the identity operator on $X$.

Theorem 3.1 ([34]) Let $X$ be a Banach space with a Schauder basis $\left(b_{k}\right)_{k=0}^{\infty}, Q \in M_{X}$, and $P_{n}: X \rightarrow X(n \in \mathbb{N})$ be the projector onto the linear span of $\left\{b_{0}, b_{1}, \ldots, b_{n}\right\}$. Then we have

$$
\frac{1}{a} \cdot \limsup _{n \rightarrow \infty}\left(\sup _{x \in Q}\left\|\left(I-P_{n}\right)(x)\right\|\right) \leq \chi(Q) \leq \limsup _{n \rightarrow \infty}\left(\sup _{x \in Q}\left\|\left(I-P_{n}\right)(x)\right\|\right),
$$

where $a=\limsup _{n \rightarrow \infty}\left\|I-P_{n}\right\|$. 
Further, the following result shows how to compute the Hausdorff measure of noncompactness in the spaces $c_{0}$ and $l_{p}(1 \leq p<\infty)$ which are $B K$-spaces with $A K$.

Theorem 3.2 ([34]) Let $X$ be the normed space that $l_{p}$ for $1 \leq p<\infty$ or $c_{0}$ and $Q$ be a bounded subset of $X$. If $P_{n}: X \rightarrow X(n \in \mathbb{N})$ is the operator defined by $P_{n}(x)=x^{[n]}=$ $\left(x_{0}, x_{1}, \ldots, x_{n}, 0,0, \ldots\right)$ for all $x=\left(x_{k}\right)_{k=0}^{\infty} \in X$, then we have

$$
\chi(Q)=\limsup _{n \rightarrow \infty}\left(\sup _{x \in Q}\left\|\left(I-P_{n}\right)(x)\right\|\right) .
$$

An infinite matrix $T=\left(t_{n k}\right)$ is called a triangle if $t_{n n} \neq 0$ and $t_{n k}=0$ for all $k>n(n \in \mathbb{N})$.

Lemma 3.1 ([33]) Let $T$ be a triangle. Then we have:

(a) For arbitrary subsets $X$ and $Y$ of $w, A \in\left(X, Y_{T}\right)$ if and only if $B=T A \in(X, Y)$.

(b) Further, if $X$ and $Y$ are $B K$-spaces and $A \in\left(X, Y_{T}\right)$, then $\left\|L_{A}\right\|=\left\|L_{B}\right\|$.

\section{Compact operators on the spaces $I_{\infty}(\tilde{B}, p), c(\tilde{B}, p)$, and $c_{0}(\tilde{B}, p)$}

In this section, we establish some identities or estimates for the Hausdorff measure of noncompactness of certain operators on the spaces $l_{\infty}(\tilde{B}, p), c(\tilde{B}, p)$, and $c_{0}(\tilde{B}, p)$. Also, we apply our results to characterize some classes of compact operators on those spaces.

We mentioned the following lemmas, which will be used in proving our results.

Lemma 4.1 ([32]) Let $X \supset \phi$ be a BK-space with $A K$ or $X=l_{\infty}$. If $A \in(X, C)$, then the following hold:

$$
\begin{aligned}
& \alpha_{k}=\lim _{n \rightarrow \infty} a_{n k} \quad \text { exists for every } k \in \mathbb{N}, \\
& \alpha=\left(\alpha_{k}\right) \in X^{\beta}, \\
& \sup _{n}\left\|A_{n}-\alpha\right\|_{X}^{*}<\infty, \\
& \lim _{n \rightarrow \infty} A_{n}(x)=\sum_{k=0}^{\infty} \alpha_{k} x_{k} \text { for all } x=\left(x_{k}\right) \in X .
\end{aligned}
$$

Lemma 4.2 ([32]) Let $X \supset \phi$ be a BK-space. Then we have:

(a) If $A \in\left(X, c_{0}\right)$, then

$$
\left\|L_{A}\right\|_{\chi}=\lim _{r \rightarrow \infty}\left(\sup _{n>r}\left\|A_{n}\right\|_{X}^{*}\right) .
$$

(b) If $X$ has $A K$ or $X=l_{\infty}$ and $A \in(X, c)$, then

$$
\frac{1}{2} \cdot \lim _{r \rightarrow \infty}\left(\sup _{n \geq r}\left\|A_{n}-\alpha\right\|_{X}^{*}\right) \leq\left\|L_{A}\right\|_{\chi} \leq \lim _{r \rightarrow \infty}\left(\sup _{n \geq r}\left\|A_{n}-\alpha\right\|_{X}^{*}\right),
$$

where $\alpha=\left(\alpha_{k}\right)$ with $\alpha_{k}=\lim _{n \rightarrow \infty} a_{n k}$ for all $k \in \mathbb{N}$.

(c) If $A \in\left(X, l_{\infty}\right)$, then

$$
0 \leq\left\|L_{A}\right\|_{\chi} \leq \lim _{r \rightarrow \infty}\left(\sup _{n>r}\left\|A_{n}\right\|_{X}^{*}\right) .
$$


Theorem 4.1 Let $X$ denote any of the spaces $l_{\infty}(\tilde{B}, p), c(\tilde{B}, p)$ or $c_{0}(\tilde{B}, p)$, and $q=\left(q_{k}\right)$ be a bounded sequence of strictly positive real numbers. Then we have:

(a) If $A \in\left(X, c_{0}(q)\right)$, then

$$
\left\|L_{A}\right\|_{\chi}=\limsup _{n \rightarrow \infty}\left(\sum_{k=0}^{\infty}\left|\tilde{a}_{n k}\right|^{p_{k}}\right)^{\frac{1}{M}}
$$

and

$$
L_{A} \text { is compact if and only if } \lim _{n \rightarrow \infty}\left(\sum_{k=0}^{\infty}\left|\tilde{a}_{n k}\right|^{p_{k}}\right)^{\frac{1}{M}}=0 \text {. }
$$

(b) If $A \in(X, c(q))$, then we have

$$
\frac{1}{2} \cdot \limsup _{n \rightarrow \infty}\left(\sum_{k=0}^{\infty}\left|\tilde{a}_{n k}-\tilde{\alpha}_{k}\right|^{p_{k}}\right)^{\frac{1}{M}} \leq\left\|L_{A}\right\|_{\chi} \leq \limsup _{n \rightarrow \infty}\left(\sum_{k=0}^{\infty}\left|\tilde{a}_{n k}-\tilde{\alpha}_{k}\right|^{p_{k}}\right)^{\frac{1}{M}}
$$

and

$$
L_{A} \text { is compact if and only if } \lim _{n \rightarrow \infty}\left(\sum_{k=0}^{\infty}\left|\tilde{a}_{n k}-\tilde{\alpha}_{k}\right|^{p_{k}}\right)^{\frac{1}{M}}=0,
$$

where $\lim _{n \rightarrow \infty} \tilde{a}_{n k}=\tilde{\alpha}_{k}$.

(c) If $A \in\left(X, l_{\infty}(q)\right)$, then

$$
0 \leq\left\|L_{A}\right\|_{\chi} \leq \limsup _{n \rightarrow \infty}\left(\sum_{k=0}^{\infty}\left|\tilde{a}_{n k}\right|^{p_{k}}\right)^{\frac{1}{M}}
$$

and

$$
L_{A} \text { is compact if and only if } \lim _{n \rightarrow \infty}\left(\sum_{k=0}^{\infty}\left|\tilde{a}_{n k}\right|^{p_{k}}\right)^{\frac{1}{M}}=0 .
$$

Proof We begin with the proof of parts (a) and (c). We know, $A \in\left(X, c_{0}(q)\right)$ if and only if $A_{n} \in X^{\beta}(\forall n \in \mathbb{N})$ and $A x \in c_{0}(p)$ for all $x \in X$. So, from Lemma 2.3

$$
\left\|A_{n}\right\|_{X}^{*}=\left\|\tilde{A}_{n}\right\|_{X^{\beta}}=\left\|\tilde{A}_{n}\right\|_{l(q)}=\sum_{k=0}^{\infty}\left|\tilde{a}_{n k}\right|
$$

for all $k \in \mathbb{N}$. Thus, we get (22) from (28) and Lemma 4.2(a). Similarly, we can get (26) from (28) and Lemma 4.2(c).

Further, if $A$ is an infinite matrix defined by

$$
a_{n k}= \begin{cases}r_{k}, & k=n \\ s_{k}, & k=n-1, \\ 0, & \text { otherwise, }\end{cases}
$$


then

$$
A x=a_{n n-1} x_{n-1} e^{n-1}+a_{n n} x_{n} e^{n}=s_{n-1} x_{n-1} e^{n-1}+r_{n} x_{n} e^{n} .
$$

So, by (11) it is easy to see that $\tilde{a}_{n k}=0$. Then $\tilde{A}_{n}=0$ and

$$
\lim _{n \rightarrow \infty}\left\|\tilde{A}_{n}\right\|_{l(q)}=\lim _{n \rightarrow \infty}\left(\sum_{k=0}^{\infty}\left|\tilde{a}_{n k}\right|^{p_{k}}\right)^{\frac{1}{M}}=0 .
$$

Hence, by combining the latter and (15) we get (23) and (27).

To prove (b) we combine Lemma 2.3 and Lemma 4.2(b) and we have (24).

We write $S=S_{X}$, for short. Then we obtain by (13) and Remark 1.1(c')

$$
\left\|L_{A}\right\|_{\chi}=\chi(A S) .
$$

So we have $A S \in M_{c(p)}$ (where $M_{c}$ is the class of all bounded subsets of $c(q)$ ). Thus, we are going to apply Theorem 3.2 to get an estimate for the value of $\chi(A S)$ in (29). To this aim, we define the projectors $P_{r}: c(q) \rightarrow c(q)(r \in \mathbb{N})$ by $P_{0}(z)=\bar{z} e$ and $P_{r}(z)=\bar{z} e+\sum_{n=0}^{r-1}\left(z_{n}-\right.$ $\bar{z}$ ) $e^{(n)}$ for $r \geq 1$ (where $z=\left(z_{k}\right) \in c(q)$ and $\left.\bar{z}=\lim _{n \rightarrow \infty} z_{n}\right)$. Then we have for every $r \in \mathbb{N}$, $\left(I-P_{r}\right)(z)=\sum_{n=r}^{\infty}\left(z_{n}-\bar{z}\right) e^{(n)}$ and hence

$$
\left\|\left(I-P_{r}\right)(z)\right\|_{l_{\infty}(p)}=\sup _{n \geq r}\left|z_{n}-\bar{z}\right|^{\frac{p_{k}}{M}}
$$

for all $z \in c(q)$ and every $r \in \mathbb{N}$. Thus, from (29) and Theorem 3.2, we get

$$
\frac{1}{2} \cdot \lim _{r \rightarrow \infty}\left(\sup _{x \in S}\left\|\left(I-P_{r}\right)(A x)\right\|_{l_{\infty}(p)}\right) \leq\left\|L_{A}\right\|_{\chi} \leq \lim _{r \rightarrow \infty}\left(\sup _{x \in S}\left\|\left(I-P_{r}\right)(A x)\right\|_{l_{\infty}(p)}\right) .
$$

Now, for every given $x \in X$, let $y \in Y$ be an associated sequence space defined by (2), where $Y$ is, respectively, the spaces $l_{\infty}(q), c(q)$ or $c_{0}(q)$. It is given that $A \in(X, c(q))$, then by Lemma 2.4 we have $\tilde{A} \in(Y, c(q))$ and $A x=\tilde{A} y$. Further, it follows from Lemma 4.1 that the limits $\tilde{\alpha}_{k}=\lim _{n \rightarrow \infty} \tilde{a}_{n k}$ exist for all $k, \tilde{\alpha}=\left(\tilde{\alpha}_{k}\right) \in l(q)=Y^{\beta}$ and $\lim _{n \rightarrow \infty} \tilde{A}_{n}(Y)=\sum_{k=0}^{\infty} \tilde{\alpha}_{k} y_{k}$. Therefore, we derive from (30) that

$$
\begin{aligned}
\left\|\left(I-P_{r}\right)(A x)\right\|_{l_{\infty}(q)} & =\left\|\left(I-P_{r}\right)(\tilde{A} y)\right\|_{l_{\infty}(q)} \\
& =\sup _{n}\left|\tilde{A}_{n}(y)-\sum_{k=0}^{\infty} \tilde{\alpha}_{k} y_{k}\right|^{\frac{p_{k}}{M}} \\
& =\sup _{n}\left|\sum_{k=0}^{\infty}\left(\tilde{a}_{n k}-\tilde{\alpha}_{k}\right) y_{k}\right|^{\frac{p_{k}}{M}}
\end{aligned}
$$

for $r \in N$. Since $x \in S=S_{X}$ if and only if $y \in S_{Y}$, we obtain by (3) and Remark 1.1(c')

$$
\begin{aligned}
\sup \left\|\left(I-P_{r}\right)(A x)\right\|_{l_{\infty}(q)} & =\sup _{n>r}\left(\sup \left|\sum_{k=0}^{\infty}\left(\tilde{a}_{n k}-\tilde{\alpha}_{k}\right) y_{k}\right|^{\frac{p_{k}}{M}}\right) \\
& =\sup _{n}\left\|\tilde{A}_{n}-\tilde{\alpha}\right\|_{Y}^{*}=\sup _{n}\left\|\tilde{A}_{n}-\tilde{\alpha}\right\|_{l(q)}
\end{aligned}
$$


for all $r \in N$. So, we obtain (24) and (25) by (31) and (14), respectively, and this completes the proof.

The following lemmas are necessary for the next theorem.

Lemma $4.3([32])$ Let $x=\left(x_{n}\right) \in l_{1}$. Then the inequalities

$$
\sup _{N \in F_{r}}\left|\sum_{n \in \mathbb{N}} x_{n}\right| \leq \sum_{n=r+1}^{\infty}\left|x_{n}\right| \leq 4 \cdot \sup _{N \in F_{r}}\left|\sum_{n \in \mathbb{N}} x_{n}\right|
$$

hold for every $r \in \mathbb{N}$.

Lemma $4.4([32])$ Let $X \supset \phi$ be a BK-space. If $A \in\left(X, l_{1}\right)$, then

$$
\lim _{r \rightarrow \infty}\left(\sup _{N \in F_{r}}\left(\left\|\sum_{n \in \mathbb{N}} A_{n}\right\|_{X}^{*}\right)\right) \leq\left\|L_{A}\right\|_{\chi} \leq 4 \cdot \lim _{r \rightarrow \infty}\left(\sup _{N \in F_{r}}\left(\left\|\sum_{n \in \mathbb{N}} A_{n}\right\|_{X}^{*}\right)\right)
$$

and

$$
L_{A} \text { is compact if and only if } \lim _{r \rightarrow \infty}\left(\sup _{N \in F_{r}}\left(\left\|\sum_{n \in \mathbb{N}} A_{n}\right\|_{X}^{*}\right)\right)=0 \text {. }
$$

Theorem 4.2 Let $X$ denote any of the spaces $l_{\infty}(\tilde{B}, p), c(\tilde{B}, p)$ or $c_{0}(\tilde{B}, p)$. If $A \in(X, l(q))$, then

$$
\lim _{r \rightarrow \infty}\left(\|A\|_{(X, l(q))}^{(r)}\right) \leq\left\|L_{A}\right\|_{\chi} \leq 4 \cdot \lim _{r \rightarrow \infty}\left(\|A\|_{(X, l(q))}^{(r)}\right)
$$

where

$$
\|A\|_{(X, l(q))}^{(r)}=\sup _{N \in F_{r}}\left(\sum_{k=0}^{\infty}\left|\sum_{n \in \mathbb{N}} \tilde{a}_{n k}\right|^{p_{k}}\right)^{\frac{1}{M}} \quad(r \in \mathbb{N})
$$

and

$$
L_{A} \text { is compact if and only if } \lim _{r \rightarrow \infty}\left(\|A\|_{(X, l(q))}^{(r)}\right)=0 \text {. }
$$

Proof Since $F \supset F_{0} \supset F_{1} \supset \ldots$, by using Remark1.2(b), the sequence $\left(\|A\|_{(X, l(q))}^{(r)}\right)_{r=0}^{\infty}$ of nonnegative reals is nonincreasing and bounded. So, the limit in (38) exists. Now, we write $S=S_{X}$ for short. Then we have $L_{A}(S)=A S \in M_{l(q)}$ by Remark 1.1(c). So, it follows from (13) and Theorem 3.2 that

$$
\left\|L_{A}\right\|_{\chi}=\chi(A S)=\lim _{r \rightarrow \infty}\left(\sup _{x \in S}\left(\sum_{n=r+1}^{\infty}\left|A_{n}(x)\right|^{p_{k}}\right)\right) .
$$

By Lemma 4.4, we have

$$
\sup _{N \in F_{r}}\left|\sum_{n \in \mathbb{N}} A_{n}(x)\right|^{p_{k}} \leq \sum_{n=r+1}^{\infty}\left|A_{n}(x)\right|^{p_{k}} \leq 4 \cdot \sup _{N \in F_{r}}\left|\sum_{n \in \mathbb{N}} A_{n}(x)\right|^{p_{k}}
$$


for all $x \in X$ and every $r \in \mathbb{N}$, because $A \in(X, l(q))$. Since $A_{n} \in X^{\beta}$ for all $n \in \mathbb{N}$, we derive from (3) and Lemma 2.3 that

$$
\begin{aligned}
\sup _{x \in S}\left|\sum_{n \in \mathbb{N}} A_{n}(x)\right|^{\frac{p_{k}}{M}} & =\sup _{x \in S}\left|\sum_{k=0}^{\infty}\left(\sum_{n \in \mathbb{N}} a_{n k}\right) x_{k}\right|^{\frac{p_{k}}{M}} \\
& =\left\|\left(\sum_{n \in \mathbb{N}} A_{n}\right)^{\frac{p_{k}}{M}}\right\|_{X}^{*} \\
& =\left\|\left(\sum_{n \in \mathbb{N}} \tilde{A}_{n}\right)^{\frac{p_{k}}{M}}\right\|_{l(q)}
\end{aligned}
$$

for all $N \in F_{r}(r \in \mathbb{N})$. These relations, together with (40), imply

$$
\begin{aligned}
\sup _{N \in F_{r}}\left\|\left(\sum_{n \in \mathbb{N}} \tilde{A}_{n}\right)^{\frac{p_{k}}{M}}\right\|_{l(q)} & \leq \sup _{x \in S}\left(\sum_{n=r+1}^{\infty}\left|A_{n}(x)\right|^{\frac{p_{k}}{M}}\right) \\
& \leq 4 \cdot \sup _{N \in F_{r}}\left\|\left(\sum_{n \in \mathbb{N}} \tilde{A}_{n}\right)^{\frac{p_{k}}{M}}\right\|_{l(q)}
\end{aligned}
$$

for every $r \in \mathbb{N}$. Thus, by passing to the limits in (41) as $r \rightarrow \infty$ and using (39), we get (38). This completes the proof.

\section{Some applications}

In this section we obtain some estimates or identities for the operator norms of certain matrix operators and we deduce the necessary and sufficient conditions for such operators to be compact. Furthermore, we obtain these results by using the Hausdorff measure of the noncompactness technique.

Corollary 5.1 Let $X$ denote any of the spaces $l_{\infty}(\tilde{B}, p), c(\tilde{B}, p)$ or $c(\tilde{B}, p)$, and $A$ be an infinite matrix. If $A$ is in any of the classes $\left(X, c s_{0}\right),(X, c s)$ or $(X, b s)$, then we have

$$
\left\|L_{A}\right\|=\sup _{n}\left(\sum_{k=0}^{\infty}\left|\sum_{m=0}^{n} \tilde{a}_{m k}\right|^{p_{k}}\right)^{\frac{1}{M}}<\infty \quad(n \in \mathbb{N}) .
$$

\section{Further:}

(a) If $A \in\left(X, c s_{0}\right)$, then

$$
\left\|L_{A}\right\|_{\chi}=\limsup _{n \rightarrow \infty}\left(\sum_{k=0}^{\infty}\left|\sum_{m=0}^{n} \tilde{a}_{m k}\right|^{p_{k}}\right)^{\frac{1}{M}}
$$

and

$$
L_{A} \text { is compact if and only if } \lim _{n \rightarrow \infty}\left(\sum_{k=0}^{\infty}\left|\sum_{m=0}^{n} \tilde{a}_{m k}\right|^{p_{k}}\right)^{\frac{1}{M}}=0 \text {. }
$$


(b) If $A \in(X, c s)$, then

$$
\begin{aligned}
& \frac{1}{2} \cdot \limsup _{n \rightarrow \infty}\left(\sum_{k=0}^{\infty}\left|\sum_{m=0}^{n} \tilde{a}_{m k}-\tilde{a}_{k}\right|^{p_{k}}\right)^{\frac{1}{M}} \\
& \quad \leq\left\|L_{A}\right\|_{\chi} \leq \limsup _{n \rightarrow \infty}\left(\sum_{k=0}^{\infty}\left|\sum_{m=0}^{n} \tilde{a}_{m k}-\tilde{a}_{k}\right|^{p_{k}}\right)^{\frac{1}{M}},
\end{aligned}
$$

where

$$
\tilde{a}=\left(\tilde{a}_{k}\right) \quad \text { with } \tilde{a}_{k}=\lim _{n \rightarrow \infty}\left(\sum_{m=0}^{n} \tilde{a}_{m k}\right) \text { for all } k \in \mathbb{N}
$$

and

$$
L_{A} \text { is compact if and only if } \lim _{n \rightarrow \infty}\left(\sum_{k=0}^{\infty}\left|\sum_{m=0}^{n} \tilde{a}_{m k}-\tilde{a}_{k}\right|^{p_{k}}\right)^{\frac{1}{M}}=0 \text {. }
$$

(c) If $A \in(X, b s)$, then

$$
0 \leq\left\|L_{A}\right\|_{\chi} \leq \limsup _{n \rightarrow \infty}\left(\sum_{k=0}^{\infty}\left|\sum_{m=0}^{n} \tilde{a}_{m k}\right|^{p_{k}}\right)^{\frac{1}{M}}
$$

and

$$
L_{A} \text { is compact if and only if } \lim _{n \rightarrow \infty}\left(\sum_{k=0}^{\infty}\left|\sum_{m=0}^{n} \tilde{a}_{m k}\right|^{p_{k}}\right)^{\frac{1}{M}}=0 \text {. }
$$

Proof The equality in (42) is immediate by Theorem 2.1. By using Theorem 4.1 in [32] in the new spaces $l_{\infty}(\tilde{B}, p), c(\tilde{B}, p)$, and $c_{0}(\tilde{B}, p)$ (for $\left.X\right)$ and by applying Lemma 2.1 and these relations with $c s_{0}=\left(c_{0}\right)_{s}, c s=(c)_{s}$, and $(b s)=\left(l_{\infty}\right)_{s}$ we obtain (43), (45), and (47). Then by using Theorem 4.2 in [32] and Lemma 2.1 we obtain (44), (46), and (48).

We denote the space of all sequences of bounded variation by $b v$, that is,

$$
b v=\left\{x=\left(x_{k}\right) \in w:\left(x_{k}-x_{k-1}\right) \in l_{1}\right\} .
$$

Corollary 5.2 Let $X$ denote any of the spaces $l_{\infty}(\tilde{B}, p), c(\tilde{B}, p)$ or $c_{0}(\tilde{B}, p)$, and $A$ be an infinite matrix. If $A \in(X, b v)$, then

$$
\begin{aligned}
& \sup _{N \in F}\left[\sum_{k=0}^{\infty}\left|\sum_{n \in \mathbb{N}}\left(\tilde{a}_{n k}-\tilde{a}_{(n-1)(k-1)}\right)^{p_{k}}\right|^{\frac{1}{M}}\right] \\
& \leq\left\|L_{A}\right\| \leq 4 \cdot \sup _{N \in F}\left[\sum_{k=0}^{\infty}\left|\sum_{n \in \mathbb{N}}\left(\tilde{a}_{n k}-\tilde{a}_{(n-1)(k-1)}\right)^{p_{k}}\right|^{\frac{1}{M}}\right] .
\end{aligned}
$$


Furthermore, if $A \in(X, b v)$, then

$$
\begin{aligned}
& \lim _{r \rightarrow \infty}\left(\sup _{N \in F_{r}}\left[\sum_{k=0}^{\infty}\left|\sum_{n \in \mathbb{N}}\left(\tilde{a}_{n k}-\tilde{a}_{(n-1)(k-1)}\right)^{p_{k}}\right|^{\frac{1}{M}}\right]\right) \\
& \leq\left\|L_{A}\right\|_{\chi} \leq 4 \cdot \lim _{r \rightarrow \infty}\left(\sup _{N \in F_{r}}\left[\sum_{k=0}^{\infty}\left|\sum_{n \in \mathbb{N}}\left(\tilde{a}_{n k}-\tilde{a}_{(n-1)(k-1)}\right)^{p_{k}}\right|^{\frac{1}{M}}\right]\right)
\end{aligned}
$$

and

$$
L_{A} \text { is compact if and only if } \lim _{r \rightarrow \infty}\left(\sup _{N \in F_{r}}\left(\sum_{k=0}^{\infty}\left|\sum_{n \in \mathbb{N}}\left(\tilde{a}_{n k}-\tilde{a}_{(n-1)(k-1)}\right)\right|^{p_{k}}\right)^{\frac{1}{M}}\right)=0 \text {. }
$$

Proof See Theorem 4.4 in [32] and Theorem 4.2.

We write $b v^{p}$ for the space of all sequences of $p$-bounded variation, that is,

$$
b v^{p}=\left\{x=\left(x_{k}\right) \in w:\left(x_{k}-x_{k-1}\right) \in l_{p}\right\} \quad(1<p<\infty) .
$$

$b v^{p}$ is a $B K$-space with its natural norm (cf. [35]). For every $a=a_{k} \in\left(b v^{p}\right)^{\beta}$, we have

$$
\|a\|_{b v^{p}}^{*}=\left(\sum_{k=0}^{\infty}\left|\sum_{j=k}^{\infty} a_{j}\right|^{q}\right)^{\frac{1}{q}}
$$

So, by using (49) we obtain the following consequence of Theorem 3.3 in [32] in the spaces $l_{\infty}(\tilde{B}, p), c(\tilde{B}, p)$, and $c_{0}(\tilde{B}, p)$.

Corollary 5.3 Let $X$ denote any of the spaces $l_{\infty}(\tilde{B}, p)$ and $c_{0}(\tilde{B}, p), 1<p<\infty, q=\left(q_{k}\right)$ be a bounded sequence of strictly positive real numbers and $A$ is an infinite matrix. If $A \in$ $\left(b v^{p}, X\right)$, then

$$
\left\|L_{A}\right\|=\|A\|_{\left(b v^{p}, l_{\infty}(\tilde{B}, p)\right)}^{(r)}=\sup _{n>r}\left(\sum_{k=0}^{\infty}\left(\left|\sum_{j=k}^{\infty} \tilde{a}_{n j}\right|^{q_{k}}\right)^{\frac{1}{q_{k}}}\right)^{\frac{p_{k}}{M}} \quad(r \in \mathbb{N}) .
$$

\section{Further:}

(a) If $A \in\left(b v^{p}, l_{\infty}(\tilde{B}, p)\right)$, then

$$
0 \leq\left\|L_{A}\right\|_{\chi} \leq \lim _{r \rightarrow \infty}\left(\sup _{n>r}\left(\sum_{k=0}^{\infty}\left|\sum_{j=k}^{\infty} \tilde{a}_{n j}\right|^{q_{k}}\right)^{\frac{1}{q_{k}}}\right)^{\frac{p_{k}}{M}}
$$

and

$$
L_{A} \text { is compact if and only if } \lim _{r \rightarrow \infty}\left(\sup _{n>r}\left(\sum_{k=0}^{\infty}\left|\sum_{j=k}^{\infty} \tilde{a}_{n j}\right|^{q_{k}}\right)^{\frac{1}{q_{k}}}\right)^{\frac{p_{k}}{M}}=0 .
$$


(b) If $A \in\left(b v^{p}, c_{0}(\tilde{B}, p)\right)$, then

$$
\left\|L_{A}\right\|_{\chi}=\lim _{r \rightarrow \infty}\left(\sup _{n>r}\left(\sum_{k=0}^{\infty}\left|\sum_{j=k}^{\infty} \tilde{a}_{n j}\right|^{q_{k}}\right)^{\frac{1}{q_{k}}}\right)^{\frac{p_{k}}{M}}
$$

and

$$
L_{A} \text { is compact if and only if } \lim _{r \rightarrow \infty}\left(\sup _{n>r}\left(\sum_{k=0}^{\infty}\left|\sum_{j=k}^{\infty} \tilde{a}_{n j}\right|^{q_{k}}\right)^{\frac{1}{q_{k}}}\right)^{\frac{p_{k}}{M}}=0 \text {. }
$$

Proof The proof is a special case of Theorem 3.3 in [32] in the new spaces $l_{\infty}(\tilde{B}, p)$ and $c_{0}(\tilde{B}, p)$ when $X=b v^{p}$. Now by using Lemma 2.1 the proof is complete.

Now, we consider some relations between $X$ (where $X$ is any of the spaces $l_{\infty}(\tilde{B}, p), c(\tilde{B}, p)$ or $\left.c_{0}(\tilde{B}, p)\right)$ and some another sequence spaces derived by the domain of the triple band matrix. First we introduce the sequence spaces $l_{\infty}(B), c(B), c_{0}(B)$, and $l_{p}(B)$ as the set of all sequences whose $B(r, s, t)$-transforms are in the spaces $l_{\infty}, c, c_{0}$, and $l_{p}$, respectively (for more details refer to [36]),

$$
\begin{aligned}
& l_{\infty}(B)=\left\{x=\left(x_{k}\right) \in w: \sup _{k \in \mathbb{N}}\left|r x_{k}+s x_{k-1}+t x_{k-2}\right|<\infty\right\}, \\
& c(B)=\left\{x=\left(x_{k}\right) \in w: \exists l \in \mathbb{C} \ni \lim \left|r x_{k}+s x_{k-1}+t x_{k-2}-l\right|=0\right\}, \\
& c_{0}(B)=\left\{x=\left(x_{k}\right) \in w: \lim \left|r x_{k}+s x_{k-1}+t x_{k-2}\right|=0\right\}, \\
& l_{p}(B)=\left\{x=\left(x_{k}\right) \in w: \sum_{k}\left|r x_{k}+s x_{k-1}+t x_{k-2}\right|^{p}<\infty\right\} .
\end{aligned}
$$

We now consider the special case of Theorems 4.1 and 4.2 in [32] when $T=B(\tilde{r}, \tilde{s})$, and $X=l_{p}(B)$.

Corollary 5.4 Let $X$ denote any of the spaces $l_{\infty}(\tilde{B}, p), c(\tilde{B}, p)$ or $c_{0}(\tilde{B}, p)$, and $A$ be an infinite matrix. If $A \in\left(l_{q}(B), X\right)$, then

$$
\left\|L_{A}\right\|=\|A\|_{\left(l_{q}(B), l_{\infty}(\tilde{B}, p)\right)}=\sup _{n}\left(\left(\sum_{k=0}^{\infty}\left|\tilde{a}_{n k}\right|^{p_{k} q_{k}}\right)^{\frac{1}{q_{k}}}\right)^{\frac{1}{M}}<\infty,
$$

where $q=\left(q_{k}\right)$ is a bounded sequence of strictly positive real numbers.

Further:

(a) If $A \in\left(l_{q}(B), c_{0}(\tilde{B}, p)\right)$, then

$$
\left\|L_{A}\right\|_{\chi}=\limsup _{n \rightarrow \infty}\left(\left(\sum_{k=0}^{\infty}\left|\tilde{a}_{n k}\right|^{p_{k} q_{k}}\right)^{\frac{1}{q_{k}}}\right)^{\frac{1}{M}}<\infty
$$

and

$$
L_{A} \text { is compact if and only if } \limsup _{n \rightarrow \infty}\left(\left(\sum_{k=0}^{\infty}\left|\tilde{a}_{n k}\right|^{p_{k} q_{k}}\right)^{\frac{1}{q_{k}}}\right)^{\frac{1}{M}}=0 .
$$


(b) If $A \in\left(l_{q}(B), c(\tilde{B}, p)\right)$, then

$$
\begin{aligned}
& \frac{1}{2} \cdot \limsup _{n \rightarrow \infty}\left(\left(\sum_{k=0}^{\infty}\left|\tilde{a}_{n k}-\tilde{\alpha}_{k}\right|^{p_{k} q_{k}}\right)^{\frac{1}{q_{k}}}\right)^{\frac{1}{M}} \\
& \quad \leq\left\|L_{A}\right\|_{\chi} \leq \limsup _{n \rightarrow \infty}\left(\left(\sum_{k=0}^{\infty}\left|\tilde{a}_{n k}-\tilde{\alpha}_{k}\right|^{p_{k} q_{k}}\right)^{\frac{1}{q_{k}}}\right)^{\frac{1}{M}},
\end{aligned}
$$

where $\tilde{\alpha}_{k}=\left(\tilde{\alpha}_{n k}\right)$ with $\lim _{n \rightarrow \infty} \tilde{a}_{n k}=\tilde{\alpha}_{k}$ and

$$
L_{A} \text { is compact if and only if } \limsup _{n \rightarrow \infty}\left(\left(\sum_{k=0}^{\infty}\left|\tilde{a}_{n k}-\tilde{\alpha}_{k}\right|^{p_{k} q_{k}}\right)^{\frac{1}{q_{k}}}\right)^{\frac{1}{M}}=0 \text {. }
$$

(c) If $A \in\left(l_{q}(B), l_{\infty}(\tilde{B}, p)\right)$, then

$$
0 \leq\left\|L_{A}\right\|_{\chi} \leq \limsup _{n \rightarrow \infty}\left(\left(\sum_{k=0}^{\infty}\left|\tilde{a}_{n k}\right|^{p_{k} q_{k}}\right)^{\frac{1}{q_{k}}}\right)^{\frac{1}{M}}<\infty
$$

and

$$
L_{A} \text { is compact if and only if } \limsup _{n \rightarrow \infty}\left(\left(\sum_{k=0}^{\infty}\left|\tilde{a}_{n k}\right|^{p_{k} q_{k}}\right)^{\frac{1}{q_{k}}}\right)^{\frac{1}{M}}=0 \text {. }
$$

Example 5.1 Let $X$ be similar to Corollary 5.4 and $Y$ be one of the spaces $c_{0}(B), c(B)$ or $l_{\infty}(B)$, and $A$ be an infinite matrix. If $A \in(X, Y)$, then

$$
\left\|L_{A}\right\|=\|A\|_{\left(X, l_{\infty}(B)\right)}=\sup _{n}\left(\sum_{k=0}^{\infty}\left|\tilde{a}_{n k}\right|^{p_{k}}\right)<\infty
$$

where $\tilde{A}=\left(\tilde{a}_{n k}\right)$ is the associated matrix defined by

$$
\tilde{a}_{n k}=\sum_{j=k}^{\infty} \sum_{i=0}^{k-j}\left(\frac{-s+\sqrt{s^{2}-4 t r}}{2 r}\right)^{j-k-i}\left(\frac{-s-\sqrt{s^{2}-4 t r}}{2 r}\right)^{i} \frac{a_{n j}}{r} .
$$

Further:

(a) If $A \in\left(X, c_{0}(B)\right)$, then

$$
\left\|L_{A}\right\|_{\chi}=\limsup _{n}\left(\sum_{k=0}^{\infty}\left|\tilde{a}_{n k}\right|^{p_{k}}\right)
$$

and

$$
L_{A} \text { is compact if and only if } \limsup _{n}\left(\sum_{k=0}^{\infty}\left|\tilde{a}_{n k}\right|^{p_{k}}\right)=0 .
$$


(b) If $A \in(X, c(B))$, then

$$
\frac{1}{2} \cdot \limsup _{n}\left(\sum_{k=0}^{\infty}\left|\tilde{a}_{n k}-\tilde{\alpha}_{k}\right|^{p_{k}}\right) \leq\left\|L_{A}\right\|_{\chi} \leq \limsup _{n}\left(\sum_{k=0}^{\infty}\left|\tilde{a}_{n k}-\tilde{\alpha}_{k}\right|^{p_{k}}\right),
$$

where $\tilde{\alpha}_{k}=\left(\tilde{\alpha}_{n k}\right)$ with $\lim _{n \rightarrow \infty} \tilde{a}_{n k}=\tilde{\alpha}_{k}$, and

$$
L_{A} \text { is compact if and only if } \underset{n}{\limsup }\left(\sum_{k=0}^{\infty}\left|\tilde{a}_{n k}-\tilde{\alpha}_{k}\right|^{p_{k}}\right)=0 .
$$

(c) If $A \in\left(X, l_{\infty}(B)\right)$, then

$$
0 \leq\left\|L_{A}\right\|_{\chi} \leq \limsup _{n}\left(\sum_{k=0}^{\infty}\left|\tilde{a}_{n k}\right|^{p_{k}}\right)
$$

and

$$
L_{A} \text { is compact if and only if } \limsup _{n}\left(\sum_{k=0}^{\infty}\left|\tilde{a}_{n k}\right|^{p_{k}}\right)=0 .
$$

Proof The proof is a consequence of Theorem 4.1.

The final result is a special case of Theorem 3.3 in [32] in the new spaces $l_{\infty}(\tilde{B}, p), c(\tilde{B}, p)$, and $c_{0}(\tilde{B}, p)$.

Corollary 5.5 Let $X$ and $Y$ be similar to Example 5.1 and $A$ be an infinite matrix. If $A \in$ $(Y, X)$, then

$$
\left\|L_{A}\right\|=\|A\|_{\left(Y, l_{\infty}(\tilde{B}, p)\right)}=\sup _{n}\left(\sum_{k=0}^{\infty}\left|\tilde{a}_{n k}\right|^{p_{k}}\right)^{\frac{1}{M}}<\infty .
$$

\section{Further:}

(a) If $A \in\left(Y, c_{0}(\tilde{B}, p)\right)$, then

$$
\left\|L_{A}\right\|_{\chi}=\limsup _{n}\left(\sum_{k=0}^{\infty}\left|\tilde{a}_{n k}\right|^{p_{k}}\right)^{\frac{1}{M}}
$$

and

$$
L_{A} \text { is compact if and only if } \limsup _{n}\left(\sum_{k=0}^{\infty}\left|\tilde{a}_{n k}\right|^{p_{k}}\right)^{\frac{1}{M}}=0 \text {. }
$$

(b) If $A \in(Y, c(\tilde{B}, p))$, then

$$
\frac{1}{2} \cdot \limsup _{n}\left(\sum_{k=0}^{\infty}\left|\tilde{a}_{n k}-\tilde{\alpha}_{k}\right|^{p_{k}}\right)^{\frac{1}{M}} \leq\left\|L_{A}\right\|_{\chi} \leq \limsup _{n}\left(\sum_{k=0}^{\infty}\left|\tilde{a}_{n k}-\tilde{\alpha}_{k}\right|^{p_{k}}\right)^{\frac{1}{M}},
$$


where $\tilde{\alpha}_{k}=\left(\tilde{\alpha}_{n k}\right)$ with $\lim _{n \rightarrow \infty} \tilde{a}_{n k}=\tilde{\alpha}_{k}$, and

$$
L_{A} \text { is compact if and only if } \limsup _{n}\left(\sum_{k=0}^{\infty}\left|\tilde{a}_{n k}-\tilde{\alpha}_{k}\right|^{p_{k}}\right)^{\frac{1}{M}}=0 \text {. }
$$

(c) If $A \in\left(Y, l_{\infty}(\tilde{B}, p)\right)$, then

$$
0 \leq\left\|L_{A}\right\|_{\chi} \leq \limsup _{n}\left(\sum_{k=0}^{\infty}\left|\tilde{a}_{n k}\right|^{p_{k}}\right)^{\frac{1}{M}}
$$

and

$$
L_{A} \text { is compact if and only if } \limsup _{n}\left(\sum_{k=0}^{\infty}\left|\tilde{a}_{n k}\right|^{p_{k}}\right)^{\frac{1}{M}}=0 \text {. }
$$

\section{Competing interests}

The authors declare that they have no competing interests.

\section{Authors' contributions}

All authors of the manuscript have read and agreed to its content and are accountable for all aspects of the accuracy and integrity of the manuscript in accordance with ICMJE criteria.

\section{Acknowledgements}

The authors would like to thank the anonymous reviewer for his/her helpful feedback and valuable suggestions, which led to an improvement in the quality of their paper.

Received: 15 August 2015 Accepted: 26 November 2015 Published online: 18 December 2015

\section{References}

1. Kuratowsky, K: Sur les espaces complets. Fundam. Math. 15, 301-309 (1930)

2. Darbo, J: Punti uniti in transformazioni a condominio non compatto. Rend. Semin. Mat. Univ. Padova 24, 84-92 (1955)

3. Goldenstein, LS, Gohberg, IT, Markus, AS: Investigations of some properties of bounded linear operators with their q-norms. Uchen. Zap. Kishinev. Gos. Univ. 29, 29-36 (1957)

4. Djolovic, I, Malkowsky, E: A note on compact operators on matrix domains. J. Math. Anal. Appl. 379, 499-511 (2011)

5. Djolovic, I, Malkowsky, E: Matrix transformations and compact operators on some new $m$ th-order difference sequences. Appl. Math. Comput. 198(2), 700-714 (2008)

6. Malkowsky, E, Racocevic, V: On matrix domains of triangles. Appl. Math. Comput. 189(2), 1146-1163 (2007)

7. Malkowsky, E, Rakocevic, $V$, Zivkovic, S: Matrix transformations between the sequence spaces $W_{0}^{p}(\Lambda), V_{0}^{p}(\Lambda), C_{0}^{p}(\Lambda)$ $(1<p<\infty)$ and certain BK-spaces. Appl. Math. Comput. 147(2), 377-396 (2004)

8. Alotaibi, A, Mursaleen, M, Alamri, BAS, Mohiuddine, SA: Compact operators on some Fibonacci difference sequence spaces. J. Inequal. Appl. 2015, 203 (2015)

9. Basar, F, Malkowsky, E: The characterization of compact operators on spaces of strongly summable and bounded sequences. Appl. Math. Comput. 217, 5199-5207 (2011)

10. Mursaleen, M, Noman, AK: Applications of the Hausdorff measure of noncompactness in some sequence spaces of weighted means. Comput. Math. Appl. 60, 1245-1258 (2010)

11. Mursaleen, M, Noman, AK: Compactness of matrix operators on some new difference sequence spaces. Linear Algebra Appl. 436, 41-52 (2012)

12. Banas, J, Mursaleen, M: Sequence Spaces and Measures of Noncompactness with Applications to Differential and Integral Equations. Springer, Berlin (2014)

13. Basarir, M, Kara, EE: On compact operators on the Riesz $B^{(m)}$ difference sequence space. Iran. J. Sci. Technol. 35(A4), 279-285 (2011)

14. Basarir, M, Kara, EE: On the $B$-difference sequence space derived by generalized weighted mean and compact operators. J. Math. Anal. Appl. 391(1), 67-81 (2012)

15. Basarir, M, Kara, EE: On the $m$ th order difference sequence space of generalized weighted mean and compact operators. Acta Math. Sci. 33(3), 797-813 (2013)

16. Ozger, F, Basar, F: Domain of the double sequential band matrix $B(\tilde{r}, \tilde{s})$ on some Maddox's spaces. Acta Math. Sci. 34(2), 394-408 (2014)

17. Kirisci, M, Basar, F: Some new sequence spaces derived by the domain of generalized difference matrix. Comput. Math. Appl. 60(5), 1299-1309 (2010)

18. Mursaleen, M, Noman, AK: On generalized means and some related sequence spaces. Comput. Math. Appl. 61(4), 988-999 (2011) 
19. Karakaya, V, Noman, AK, Polat, H: On paranormed $\lambda$-sequence spaces of non-absolute type. Math. Comput. Model. 54, 1473-1481 (2011)

20. Maddox, IJ: Some properties of paranormed sequence spaces. J. Lond. Math. Soc. 1(2), 316-322 (1969)

21. Mursaleen, $M$, Noman, AK: Hausdorff measure of noncompactness of certain matrix operators on the sequence spaces of generalized means. J. Math. Anal. Appl. 417, 96-111 (2014)

22. Malkowsky, E: Modern functional analysis in the theory of sequence spaces and matrix transformations. Jordan J. Math. Stat. 1(1), 1-29 (2008)

23. Alotaibi, A, Malkowsky, E, Mursaleen, M: Measure of noncompactness for compact matrix operators on some BK-spaces. Filomat 28, 1081-1086 (2014)

24. Maddox, IJ: Paranormed sequence spaces generated by infinite matrices. Proc. Camb. Philos. Soc. 64, 335-340 (1968)

25. Mursaleen, M, Noman, AK: The Hausdorff measure of noncompactness of matrix operators on some BK-spaces. Oper. Matrices 5(3), 473-486 (2011)

26. Mursaleen, M, Noman, AK: Applications of Hausdorff measure of noncompactness in the spaces of generalized means. Math. Inequal. Appl. 16, 207-220 (2013)

27. Basarir, M, Kara, EE: On compact operators on the Riesz $B^{(m)}$-difference sequence spaces II. Iran. J. Sci. Technol. 36(A3), 371-376 (2012)

28. Mursaleen, M, Karakaya, $V$, Polat, $H$, Simsek, N: Measure of noncompactness of matrix operators on some difference sequence spaces of weighted means. Comput. Math. Appl. 62, 814-820 (2011)

29. Kara, EE, Basarir, M: On some Euler $B^{(m)}$-difference sequence spaces and compact operators. J. Math. Anal. Appl. 379 499-511 (2011)

30. Maji, A, Srivastava, PD: On $B^{(m)}$-difference sequence spaces using generalized means and compact operators. Analysis 34(3), 257-281 (2014)

31. Kara, EE, Basarir, M, Konca, S: On some new weighted Euler sequence spaces and compact operators. Math. Inequal. Appl. 17(2), 649-664 (2014)

32. Mursaleen, M, Noman, AK: Compactness by the Hausdorff measure of noncompactness. Nonlinear Anal. 73, 2541-2557 (2010)

33. Malkowsky, E, Rakocevic, V: An introduction into the theory of sequence spaces and measure of noncompactness. Zb. Rad. (Beogr.) 9(17), 143-234 (2000)

34. Banas, J, Goeble, K: Measures of Noncompactness in Banach Spaces. Lecture Notes in Pure and Applied Mathematics, vol. 60. Dekker, New York (1980)

35. Malkowsky, E, Rakocevic, V, Zivkovic, S: Matrix transformations between the sequence spaces $b v^{p}$ and certain BK-spaces. Bull. - Acad. Serbe Sci. Arts, Cl. Sci. Math. Nat., Sci. Math. 123(27), 33-46 (2002)

36. Sonmez, A: Some new sequence spaces derived by the domain of the triple band matrix. Comput. Math. Appl. 62, 641-650 (2011)

\section{Submit your manuscript to a SpringerOpen ${ }^{\circ}$ journal and benefit from:}

- Convenient online submission

Rigorous peer review

- Immediate publication on acceptance

- Open access: articles freely available online

- High visibility within the field

- Retaining the copyright to your article 